\title{
Scaling of elongation transition thickness during thin-film growth on weakly interacting substrates
}

Bo Lü, Laurent Souqui, Viktor Elofsson and Kostas Sarakinos

The self-archived postprint version of this journal article is available at Linköping University Institutional Repository (DiVA):

http:/ / urn.kb.se/ resolve?urn=urn:nbn:se:liu:diva-140966

N.B.: When citing this work, cite the original publication.

Lü, Bo, Souqui, L., Elofsson, V., Sarakinos, K., (2017), Scaling of elongation transition thickness during thin-film growth on weakly interacting substrates, Applied Physics Letters, 111(8).

https:// doi.org/ 10.1063/ 1.4993252

Original publication available at:

https:/ / doi.org/ 10.1063/ 1.4993252

Copyright: AIP Publishing

http:// www.aip.org/ 


\title{
Scaling of elongation transition thickness during thin-film growth on weakly-interacting substrates
}

B. Lü ${ }^{1}$, L. Souquii ${ }^{1,2}$, V. Elofsson ${ }^{1}$ and K. Sarakinos ${ }^{1^{*}}$

*Corresponding author, email: kostas.sarakinos@liu.se

${ }^{1}$ Nanoscale Engineering Division, Department of Physics, Chemistry and Biology, Linköping University, SE 581 83, Linköping, Sweden

2Present address: Thin Film Physics Division, Department of Physics, Chemistry and Biology, Linköping University, SE 581 83, Linköping, Sweden

\begin{abstract}
The elongation transition thickness $\left(\theta_{\text {Elong }}\right)$ is a central concept in the theoretical description of thin-film growth dynamics on weakly-interacting substrates via scaling relations of $\theta_{\text {Elong }}$ with respect to rates of key atomistic film-forming processes. To date, these scaling laws have only been confirmed quantitatively by simulations, while experimental proof has been left ambiguous as it has not been possible to measure $\theta_{\text {Elong }}$. Here, we present a method for determining experimentally $\theta_{\text {Elong }}$ for Ag films growing on amorphous $\mathrm{SiO}_{2}$; an archetypical weakly-interacting film/substrate system. Our results confirm the theoretically predicted $\theta_{\text {Elong }}$ scaling behavior, which then allow us to calculate rates of adatom diffusion and island coalescence completion in good agreement with the literature. The methodology presented herein casts the foundation for studying growth dynamics and cataloging atomistic-process rates for a wide range of weakly-interacting films/substrate systems. This may provide insights into directed
\end{abstract}


growth of metal films with well-controlled morphology and interfacial structure on 2D crystals_-including graphene and $\mathrm{MoS}_{2}$ - for catalytic and nanoelectronic applications.

Thin-film growth on weakly-interacting substrates is of great technological importance for the field of catalysis [1], architectural glazing [2] and microelectronics. [3-5] Upon condensation on a weakly-interacting surface, film-forming species self-assemble in dispersed 3D atomic islands, which impinge on each other as they grow in size. [6] This initiates a dynamic competition between island growth and coalescence leading to the so-called elongation transition; the point during growth at which the surface is predominantly covered by elongated structures. [6] This transition is crucial in determining the resulting film microstructure, while the scaling behavior of the thickness at which it occurs, $\theta_{\text {Elong }}$, with respect to the rates of vapor deposition $F[$ monolayers $/ s]$, adatom surface diffusion $D\left[\mathrm{~cm}^{2} / s\right]$ and island coalescence completion $B\left[\mathrm{~cm}^{4} / \mathrm{s}\right]$ reflects the dynamics of early film-formation stages. [7-11] Depending on the relative rates of island growth and coalescence, two morphological evolution regimes can be established in which merging islands can either fully relax into a compact shape and thereby complete coalescence (coalescence-controlled regime) or not (coalescence-free regime). [12-16] Analytical models and growth simulations $[12,13,17-19]$ have shown that in these two regimes the $\theta_{\text {Elong }}$ scaling laws under a continuous deposition flux read, $[6,12,13]$

$$
\begin{gathered}
\theta_{\text {Elong }} \approx(1.75 \alpha B /(F \Omega))^{1 / 3}(\text { coalescence }- \text { controlled }), \\
\theta_{\text {Elong }} \approx 2\left(\frac{D}{F}\right)^{\frac{1}{7}}(\text { coalescence }- \text { free }) .
\end{gathered}
$$


In Eq. (1), $\alpha$ is the height-to-radius ratio of the islands and $\Omega\left[\mathrm{cm}^{3}\right]$ is the atomic volume of the film species. If hemispherical islands are assumed, then $\alpha=1$, while all scales can be normalized such that $\Omega=1$.

To date, $\theta_{\text {Elong }}$ has not been determined experimentally, as the elongation transition is an intrinsically abstract concept if approached directly, e.g., by visual inspection of the growth surface by scanning probe microscopies. [20,21] This casts doubt on Eqs. (1) and (2), since their experimental validation has been sought by measuring later transition thicknesses, such as the thickness at percolation $\left(\theta_{\text {Perc }}\right)$ and continuous film formation $\left(\theta_{\text {cont }}\right)$ as functions of $F$, assuming that all transition thicknesses exhibit the same scaling behavior $\theta_{\text {Cont/Perc/Elong }} \sim F^{\chi}$. Available experimental data based on $\theta_{\text {Cont }}$ seemingly confirm Eq. (2), [14,16] while $\theta_{\text {Perc }}$ and $\theta_{\text {Cont }}$ values obtained for coalescence-controlled growth at room temperature have consistently shown exponent values smaller than the theoretically predicted -1/3. [14-16,22]

Here, we resolve this ambiguity and confirm the validity of Eqs. (1) and (2) by demonstrating a method to determine $\theta_{\text {Elong }}$. This is done by depositing films in two parts, the template and top layer, with different deposition rates, $F_{\text {Temp }}$ and $F_{\text {Top }}$, respectively. The change of deposition rate from $F_{T e m p}$ to $F_{T o p}$ is made abruptly during the early-growth stages aiming to disrupt the growth evolution and thereby either activate or freeze coalescence of an established growth surface. This then affects how $\theta_{\text {Elong }}$ is set, and by association also $\theta_{\text {Cont } / \text { Perc }} \cdot[6,1319,23]$ Since past the elongation transition, other processes (e.g., hole filling and grain growth) become dominant in determining microstructural evolution, $[20,24]$ a disruption of growth dynamics made after $\theta_{\text {Elong }}$ will set $\theta_{\text {Cont/Perc }}$ in a distinctly different way as compared to a disruption 
made before. Hence, if $\theta_{\text {Cont } / \text { Perc }}$ is measured for a set of abrupt $F$-changes made at different times during growth, $\theta_{\text {Elong }}$ can be determined by identifying the point which separates two distinct behaviors or regions of $\theta_{\text {Cont/Perc }}$. Repeating this for multiple deposition rates will then generate a set of $\theta_{\text {Elong }}$ values in both the coalescencecontrolled and coalescence-free growth regimes, thus providing the means to investigate the experimental scaling behavior of $\theta_{\text {Elong }}$ with $F$ and compare it to Eqs. (1) and (2).

The concept described above was used to determine $\theta_{\text {Elong }}$ for Ag grown on $\mathrm{SiO}_{2}$ by measuring $\theta_{\text {Cont }}$ in corresponding templated films. The choice of film and substrate materials was based on their suitability as representative weakly-interacting system, as well as the availability of experimental data on the scaling behavior of $\theta_{\text {Perc }}$ and $\theta_{\text {Cont }} \cdot[14,16,22]$ The range of the template layer thickness $\left(\theta_{\text {Temp }}\right)$ was selected to be in a region where the elongation transition is expected to occur $(21-85 M L)$ based on previous experimental $\theta_{\text {Perc }}$ and $\theta_{\text {Cont }}$ data. [16,22] Films consisting of template and top layers were grown from the vapor phase by magnetron sputtering at UHV conditions (base pressure $1.3 \cdot 10^{-6} \mathrm{~Pa}$ ) at a working pressure of $0.67 \mathrm{~Pa}$, achieved by using 99.9997\% purity Ar sputtering gas. Depositions were performed on Si substrates $(10 \times 10 \mathrm{~mm})$ covered by a $5000 \AA$ thermally grown amorphous $\mathrm{SiO}_{2}$. The substrates were prepared by ultrasonic cleaning in acetone and isopropanol for 10 minutes each. No temperature control was applied to the substrates during deposition, i.e., growth at room temperature is assumed. For the depositions, pulsed vapor fluxes were used as they allow to identify the two coalescence-based growth regimes easier as compared to the case when deposition is performed using continuous vapor fluxes. [14-16,22] Vapor was generated by applying power to the sputtering source at constant energy 
per pulse of $\sim 20 \mathrm{~mJ}$ and a constant pulse width of $50 \mu \mathrm{s}$ resulting in a constant instantaneous deposition rate $F_{i}=5.68 \mathrm{ML} / \mathrm{s}$, while the pulse frequency $f$ [Hz] was varied from 100 to $1000 \mathrm{~Hz}$, yielding time averaged deposition rates from 0.04 to 0.6 ML/s, respectively. [25] This flux temporal profile leads to growth in the fast diffusion nucleation regime, in which the island saturation density $\left(N_{\text {sat }}\right)$ is independent of $F$ and scales instead with $F_{i}$. $[15,26]$ As a consequence, for coalescence-free growth $\theta_{\text {Elong }}$ scales as $\left(\frac{D}{F_{i}}\right)^{\frac{1}{7}}$ which means that for the conditions used here a constant $\theta_{\text {elong }}$ value should be obtained. [27] In addition, the chosen deposition conditions yield low energies of the sputtered species independent of $f,[25]$ which along with the fact that the substrate was held at floating potential minimizes the effects of energetic bombardment on the growing film commonly associated with pulsed deposition processes. The growth process was monitored in real time using in situ spectroscopic ellipsometry (M-88, J. A. Woollam Inc. apparatus), and a three-phase model consisting of substrate, film and vacuum was used to fit the acquired data and determine $\theta_{\text {cont }}$ as detailed in previous works. [22,25,28]

Figure 1 (a) shows $\theta_{\text {Cont }}$ as function of $\theta_{\text {Temp }}$ for different $F_{T e m p}$ when $F_{T o p} \approx 0.6 \mathrm{ML} / \mathrm{s}$, while similar data but for $F_{\text {Top }} \approx 0.04 \mathrm{ML} / \mathrm{s}$ are shown in Fig. 1 (b). In line with previous studies $[16,22]$, change of the deposition rate from $F_{\text {Temp }}$ to $F_{T o p}$ for the data in Fig. 1

(a) triggers transition from coalescence-free to coalescence-controlled growth, while the opposite transition is triggered for the data in Fig. 1 (b). In Fig. 1 (a), $\theta_{\text {cont }}$ increases gradually with increasing template thickness and reaches local maxima (indicated by the vertical dashed lines) close to $\theta_{\text {cont }}$ (horizontal dashed lines) for monolithic films grown at the corresponding $F=F_{T e m p}$. In Fig. 1 (b), $\theta_{\text {Cont }}$ decreases steeply with $\theta_{T e m p}$ to reach local minima (marked by vertical dash-dotted lines) just above the 
corresponding $\theta_{\text {cont }}$ of monolithic films. Note that this is assumed also for the case of $F_{\text {Temp }}=0.28 \mathrm{ML} / \mathrm{s}$ (black squares in Fig. 1 (b)), even though an explicit minimum was not obtained in this particular dataset. Based on the proximity of the minima in the other two datasets to their corresponding monolithic film $\theta_{\text {cont }}$, it can be estimated that the actual minimum for this dataset is within $\pm 4 M L$ of the lowest value $(72.5 \mathrm{ML})$. The template thicknesses at the local maxima and minima in Figs. 1 (a) and (b) are considered to be the $\theta_{\text {Temp }}$ values at which $\theta_{\text {cont }}$ undergoes a change in behavior as a function of $\theta_{T e m p}$, i.e., they correspond to $\theta_{\text {Elong }}$ values for each specific deposition rate.

Figure 1 (c) summarizes the measured $\theta_{\text {cont }}$ values for monolithic films along with $\theta_{\text {Elong }}$ extracted from templated films over the deposition rate range $0.04-0.6 \mathrm{ML} / \mathrm{s}$. Two power laws are identified for $\theta_{\text {Cont }}$ with scaling exponents -0.23 (presumably coalescence-controlled growth) and -0.05 (presumably coalescence-free growth) for $F$ in the range $0.04-0.17 \mathrm{ML} / \mathrm{s}$ and $0.17-0.6 \mathrm{ML} / \mathrm{s}$, respectively. The scaling exponents for $\theta_{\text {Elong }}$ in the corresponding growth regimes are found to be -0.30 and -0.05 , for $F$ in the ranges $0.04-0.1 \mathrm{ML} / \mathrm{s}$ and $0.1-0.6 \mathrm{ML} / \mathrm{s}$, respectively. To put our measurements into perspective, our data is also compared to $\theta_{\text {perc }}$ values from an independent and unrelated study of $\mathrm{Ag}$ deposited on $\mathrm{SiO}_{2}$ by pulsed laser deposition at $313 \mathrm{~K}$ (red, filled circles in Fig. 1 (c)). [16] It can be seen that the three types of transition thicknesses appear in the expected order of occurrence, beginning with elongation at the lowest thicknesses to percolation and finally continuous film formation. Moreover, the transition between coalescence-controlled and coalescence-free growth modes occur at nearly the same critical $F$ value (in the range $0.10-0.17 \mathrm{ML} / \mathrm{s}$ ) for all three transition thicknesses. These two facts embody one of the main concepts in the theoretical 
description of film growth on weakly-interacting substrates, namely that morphological features are predominantly set at the elongation transition.

To understand the origin of the features observed in the experimental results, a previously developed kinetic Monte Carlo (kMC) code was used to model our method. [14-18] This code treats nucleation, growth, impingement and coalescence of 3D hemispherical islands in terms of the parameters $F, D$, and $B . \theta_{\text {Elong }}$ is then determined by finding the film thickness at which the average number of islands in coalescing clusters exceed two. In the framework of the present study, simulations were performed using the values $D=4.39 \times 10^{-10} \mathrm{~cm}^{2} / \mathrm{s}$ and $B=3.49 \times 10^{-28} \mathrm{~cm}^{4} / \mathrm{s}$, in line with our previous studies to reproduce the two coalescence growth regimes. $[15,22]$ Abrupt changes of $F$ at various growth stages were simulated by using pulsed atomic fluxes with instantaneous rate $F_{i}=250 \mathrm{ML} / \mathrm{s}$, pulse width $100 \mu \mathrm{s}$ and two pulsing frequencies of $1000 \mathrm{~Hz}$ and $10 \mathrm{~Hz}$ leading to the respective rates $F_{1}=25 \mathrm{ML} / \mathrm{s}$ and $F_{2}=0.25 \mathrm{ML} / \mathrm{s}$, resulting in coalescence-free and coalescence-controlled growth, respectively. In a first set of simulations, growth commenced by depositing a template layer at a rate $F_{1}$, followed immediately by a top layer deposited at a rate $F_{2}$. In a second simulations set, $F_{2}$ was used as the template layer deposition rate, while the top layer was deposited at $F_{1}$. In both cases, $\theta_{\text {Elong }}$ as function of the template layer thickness was calculated, while the evolution of island density versus nominal thickness, $N(\theta)$, was also tracked. For the sake of brevity, the notation $F_{T e m p} / F_{T o p}$ is used to denote the template and top layer deposition rates when presenting the simulation results.

Figure 2 shows the evolution of $\theta_{\text {Elong }}$ as a function of $\theta_{\text {Temp }}$ for the two datasets with $F_{1} / F_{2}$ (black squares) and $F_{2} / F_{1}$ (read circles). The horizontal dash-dotted lines indicate 
$\theta_{\text {Elong }}\left(F_{1}\right)=1.02 M L$ and $\theta_{\text {Elong }}\left(F_{2}\right)=3.3 M L$ for non-templated growth (i.e., a monolithic film). For the dataset $F_{1} / F_{2}$, three distinct regions of $\theta_{\text {Elong }}$ can be identified as opposed to only two that were found in the experimental results. These are comprised of a region (I) of constant $\theta_{\text {Elong }}\left(F_{1} / F_{2}\right) \approx \theta_{\text {Elong }}\left(F_{2}\right)$ up to $\theta_{\text {Temp }}=1 M L$, followed by a narrow region (II) of sharp decline which ends in a region (III) of constant $\theta_{\text {Elong }}\left(F_{1} / F_{2}\right) \approx$ $\theta_{\text {Elong }}\left(F_{1}\right)$ for $\theta_{\text {Temp }} \geq 1.25 \mathrm{ML}$. Similarly, dataset $F_{2} / F_{1}$ also shows three different regions of $\theta_{\text {Elong }}$ : a very narrow region $(\mathrm{I})$ where $\theta_{\text {Elong }}\left(F_{2} / F_{1}\right) \approx \theta_{\text {Elong }}\left(F_{1}\right)$ for $\theta_{\text {Temp }} \leq 0.5 M L$, followed by a wide region (II) where $\theta_{\text {Elong }}$ increases until $\theta_{T e m p}=3.5 M L$, after which a narrow region (III) concludes the dataset with $\theta_{\text {Elong }}\left(F_{2} / F_{1}\right) \approx \theta_{\text {Elong }}\left(F_{2}\right)$. By comparing dataset $F_{2} / F_{1}$ with Fig. 1 (a), and dataset $F_{1} / F_{2}$ with Fig. 1 (b), we conclude that the experimental results correspond to region (II) followed by region (III) in both cases. The physical significance of the width difference of region (II) for the two datasets in Fig. 2 can be explained by plotting the island density evolution $N$ as a function of the nominal film thickness $\theta$, as in the inset of Fig. 2 for the points marked $A$ and B. For point $A$, the decrease of $F$ activates coalescence; this is what produces the sharp drop of $N$ at $\theta=$ $0.75 M L$. Thus for $F_{1} / F_{2}$, whenever $F$ is changed before elongation has been reached, the entire surface restructures to a morphology similar to that of the monolithic film grown at $F_{2}$, which in turn implies that $\theta_{\text {Elong }}$ always turns out as $\theta_{\text {Elong }}\left(F_{2}\right)$. Hence, we only see a change in $\theta_{\text {Elong }}$ for this dataset in Fig. 2 when $\theta_{\text {Temp }}$ exceeds $\theta_{\text {Elong }}\left(F_{1}\right)$, and so region (II) should ideally be infinitesimally narrow. For point B, the freezing of coalescence by increasing $F$ is seen at $\theta=2 M L$, where $N$ suddenly levels out with a slightly positive slope, and the elongation transition is reached shortly thereafter. This means that for the dataset $F_{2} / F_{1}$, when $F$ is increased before elongation is reached, $\theta_{\text {Elong }}$ is a linear function of $N\left(\theta_{\text {Temp }}\right)$, and the width of region (II) depends on how well $\theta_{\text {Elong }}\left(F_{1}\right)$ and $\theta_{\text {Elong }}\left(F_{2}\right)$ are separated. 
Our experimental results for $\theta_{\text {Elong }}$ yield a scaling exponent of -0.30 in the coalescencecontrolled growth regime which is very close to the theoretical value of -0.33 . This indicates that Eq. (1) indeed gives an accurate account of 3D island nucleation and growth dynamics with active coalescence, over the measured range of deposition rates. Moreover, the slope of $\theta_{\text {cont }}$ found in Fig. 1 (c) for coalescence-controlled growth $(-0.23)$ is in good agreement with previously published exponents in the range -0.18 to -0.25. [22] Since the scaling exponent on $\theta_{\text {cont }}$ is systematically lower than that for $\theta_{\text {Elong }}$, it is possible that processes occurring after elongation (hole-filling and grain growth) are affecting the scaling behavior of $\theta_{\text {cont }}$. For the coalescence-free growth regime, the results in Fig. 1 (c) are in close agreement with the scaling behavior $\theta_{\text {Elong }}=$ constant, i.e., 3D island nucleation and growth theory (cf. Eq. (2)) describes growth in this regime as well. However, the scaling exponents for all transition thicknesses in Fig. 1 (c) $\left(\theta_{\text {Elong }}, \theta_{\text {Perc }}\right.$ and $\left.\theta_{\text {Cont }}\right)$ in this growth regime reveal a small yet consistent dependence on $F$. Since the prediction $\theta_{\text {Elong }} \propto F^{0}$ is based on irreversible nucleation with adatoms as the only mobile species, we suggest that such a small $F$ dependence may be caused by detachment of adatoms $[29,30]$ or the presence of island mobility and Smoluchowski ripening. [31] Both of these would affect the nucleation density directly, which in turn affects $\theta_{\text {Elong }}$ indirectly through the relation $\theta_{\text {Elong }} \propto N_{\text {sat }}^{-1 / 2}$. [15] Alternatively, this could also be caused by a change of the 3D aspect ratio—a flattening out—of the islands at high deposition rates; [32] the flatter the islands, the more they spread out and the earlier elongation would occur.

Having direct measurements of $\theta_{\text {Elong }}$, Eqs. (1) and (2) can be used to provide estimates for $D$ and $B$ in the $\mathrm{Ag}-\mathrm{SiO}_{2}$ material system, as such values are sparsely 
given in the literature. Setting Eq. (1) to be equal to the curve fit expression of $\theta_{\text {Elong }}$ in the coalescence-controlled growth regime from Fig. 1 (c) yields

$$
B=C_{1}^{3} F^{3 \chi_{1}+1} \Omega /(1.75 \alpha) .
$$

Note that in the ideal case, $\chi_{1}=-1 / 3$ and there should be no $F$-dependence in Eq. (3). Thus, our results $C_{1} \approx 72.26$ and $\chi_{1}=-0.30$ permit to find for the range of $F=0.04-$ $0.1 \mathrm{ML} / \mathrm{s}$ an average $B=1.82 \times 10^{-27} \mathrm{~cm}^{4} / \mathrm{s}$. This value compares favorably with a previous estimate placing $B$ in the range $1.58 \times 10^{-28} \mathrm{~cm}^{4} / \mathrm{s}-1.22 \times 10^{-27} \mathrm{~cm}^{4} / \mathrm{s}$, based on annealing of percolated Ag films. [33] To calculate $D$, we may apply a similar procedure to the pulsed flux analogue of Eq. (2) for the fast nucleation regime,

$$
D=F_{i}\left(\left\langle\theta_{\text {Elong }}\right\rangle / 2\right)^{7}
$$

where $\left\langle\theta_{\text {Elong }}\right\rangle$ denotes an average of the values in the coalescence free growth mode. With $F_{i}=5.68 M L / s$ in our experiments and $\left\langle\theta_{\text {Elong }}\right\rangle \approx 44 M L, D$ is found to be $7.88 \times 10^{-6} \mathrm{~cm}^{2} / \mathrm{s}$. The $D$ obtained using Eq. (4) can be used to calculate an effective diffusion energy barrier $E_{D}$ for $\mathrm{Ag}$ on $\mathrm{SiO}_{2}$ from the Arrhenius equation. Using $a=2.89 \AA$ as the minimum translation distance on a $\mathrm{Ag}(111)$ surface (here assumed also for the $\mathrm{SiO}_{2}$ surface), $v_{0}$ an attempt frequency on the order of $10^{12}-10^{13} \mathrm{~Hz}$, and $\mathrm{T}=300 \mathrm{~K}$ yields $E_{D, A g / S_{2}}=0.085-0.144 \mathrm{eV}$. An early nucleation study of $\mathrm{Ag}$ on $\mathrm{SiO}_{2}$ estimates $E_{D, A g / \mathrm{SiO}_{2}} \approx 0.1 \mathrm{eV}$, although this value is considered highly approximate owing to the low vacuum quality available at the beginning of the 1970's. [34,35] A much later study makes a qualitative comparison of island densities at a fixed coverage on $\mathrm{Ag} / \mathrm{SiO}_{2}$ and $\mathrm{Ag} / \mathrm{KBr}$, both deposited under identical conditions. [36] $E_{D, A g / K B r}$ is known to be about $0.19 \mathrm{eV},[37]$ and it is argued that since the island density seen for $\mathrm{Ag} / \mathrm{SiO}_{2}$ appears to be lower than for $\mathrm{Ag} / \mathrm{KBr}$, the adatom diffusion barrier for $\mathrm{Ag} / \mathrm{SiO}_{2}$ should also be lower than $0.19 \mathrm{eV}$. Finally, a more recent study used molecular dynamics to calculate $E_{D, \mathrm{Ag} / \mathrm{SiO}_{2}} \approx 0.2 \mathrm{eV} .[38,39]$ Together, these provide an estimated range for $E_{D, \mathrm{Ag} / \mathrm{SiO}_{2}}$ 
between $0.1-0.2 \mathrm{eV}$. The present results indicate that $E_{D, \mathrm{Ag} / \mathrm{SiO}_{2}}$ is more likely to be at the lower end of this range, i.e., that $\mathrm{Ag}$ is highly mobile on $\mathrm{SiO}_{2}$, with a rate comparable to $\mathrm{Ag} / \mathrm{Ag}(111)$ surface self-diffusion $(0.067-0.097 \mathrm{eV}[40,41])$.

In this letter, we described a method to experimentally determine the elongation transition film thickness and verified this method for deposition of pulsed Ag vapor on $\mathrm{SiO}_{2}$. Experimental data in the deposition rate range $0.04-0.6 \mathrm{ML} / \mathrm{s}$ for depositions performed at room temperature reveal the existence of two scaling exponents $\chi=$ -0.30 and $\chi \approx-0.05$ for $\theta_{\text {Elong }} \propto F^{\chi}$, corresponding to coalescence-controlled and coalescence-free growth respectively. These exponents compare favorably with their theoretical predictions, $\chi=-1 / 3$ (coalescence-controlled) and $\chi=0$ (coalescencefree), thereby resolving the ambiguity regarding the validity of Eqs. (1) and (2) to describe early morphological evolution of metals on weakly-interacting substrates. Hence, Eqs. (1) and (2) can be used as a solid frame of reference to fundamentally understand the dynamics of 3D island formation and growth on weakly-interacting substrates. Using the determined values of $\theta_{\text {Elong }}$, an estimate of the coalescence rate parameter $B=1.82 \times 10^{-27} \mathrm{~cm}^{4} / \mathrm{s}$ and the adatom diffusion barrier $E_{D, A g / \mathrm{SiO}_{2}}=0.085-$ $0.144 \mathrm{eV}$ were calculated; both in good agreement with available literature values. Since experimental values of material properties (e.g. diffusion barriers) are lacking for many weakly-interacting film/substrate systems, the herein presented methodology could prove useful in the collection of such data to further explore the generality of the theoretical framework of 3D nucleation and growth beyond the model system $\left(\mathrm{Ag} / \mathrm{SiO}_{2}\right)$ used in this study. In practical terms, knowledge of the adatom diffusivity and coalescence rates, could facilitate more informed decisions regarding growth manipulation by, e.g., doping, surfactant action or tuning of the deposition rate and 
growth temperature. This may be of relevance for a number of applications such as architectural glazing, [1] microelectronics, catalysis [2] and the metallization of 2D crystals, including graphene and $\mathrm{MoS}_{2}$. [3-5]

\section{Acknowledgements}

The authors should like to acknowledge Linköping University (LiU) for financial support through the "LiU Research Fellows Program, 2011-2015" and the "LiU Career Contract (Dnr-LiU-2015-01510), 2015-2020". The study was also supported by the Swedish research council through the contracts VR-2011-5312 and VR-2015-04630. Simulations were performed using supercomputer resources provided by the Swedish National Infrastructure for Computing (SNIC) at the National Supercomputer Centre (NSC). Prof. Hans Arwin and Prof. Kenneth Järrendahl (Applied Optics Division, Linköping University) are gratefully acknowledged for providing the in situ spectroscopic ellipsometer apparatus. The authors wish to acknowledge Dr. Mattias Samuelsson (Linköping University) for his comments on the manuscript.

\section{References}

[1] C. T. Campbell, J. Chem. Soc. Faraday Trans. 92, 1435 (1996).

[2] C. Schaefer, G. Bräuer, and J. Szczyrbowski, Surf. Coatings Technol. 93, 37 (1997).

[3] X. Liu, Y. Han, J. W. Evans, A. K. Engstfeld, R. J. Behm, M. C. Tringides, M.

Hupalo, H. Q. Lin, L. Huang, K. M. Ho, D. Appy, P. A. Thiel, and C. Z. Wang, Prog.

Surf. Sci. 90, 397 (2015).

[4] C. Gong, C. Huang, J. Miller, L. Cheng, Y. Hao, D. Cobden, J. Kim, R.S. Ruoff, R.M. Wallace,K. Cho, X. Xu, Y.J. Chabal, ACS Nano 7, 11350 (2013). 
[5] Wissam A. Saidi, Crystal Growth and Design 15, 3190 (2015).

[6] G. Jeffers, M. A. Dubson, and P. M. Duxbury, J. Appl. Phys. 75, 5016 (1994).

[7] F. A. Nichols, J. Appl. Phys. 37, 2805 (1966).

[8] W. W. Mullins, J. Appl. Phys. 28, 333 (1957).

[9] C. Herring, J. Appl. Phys. 21, 301 (1950).

[10] G. C. Kuczynski, AIME Met. Trans. 185, 169 (1949).

[11] A. D. Brailsford and N. A. Gjostein, J. Appl. Phys. 46, 2390 (1975).

[12] P. Jensen, H. Larralde, M. Meunier, and A. Pimpinelli, Surf. Sci. 412/413, 458 (1998).

[13] J. Carrey and J.-L. Maurice, Phys. Rev. B 65, 205401 (2002).

[14] B. Lü, V. Elofsson, E. P. Münger, and K. Sarakinos, Appl. Phys. Lett. 105, 163107 (2014).

[15] B. Lü, E. P. Münger, and K. Sarakinos, J. Appl. Phys. 117, 134304 (2015).

[16] J. M. Warrender and M. J. Aziz, Phys. Rev. B 76, 045414 (2007).

[17] J. Carrey and J.-L. Maurice, Phys. Rev. B 63, 245408 (2001).

[18] M. Tomellini and M. Fanfoni, Phys. Rev. B 72, 155407 (2005).

[19] M. Fanfoni and M. Tomellini, Phys. Rev. B. Condens. Matter 54, 9828 (1996).

[20] C. Polop, C. Rosiepen, S. Bleikamp, R. Drese, J. Mayer, A. Dimyati, and T.

Michely, New J. Phys. 9, 74 (2007).

[21] O. Dulub, L. A. Boatner, and U. Diebold, Surf. Sci. 504, 271 (2002).

[22] V. Elofsson, B. Lü, D. Magnfält, E. P. Münger, and K. Sarakinos, J. Appl. Phys. 116, 44302 (2014).

[23] F. Ruffino and M. G. Grimaldi, J. Appl. Phys. 107, 74301 (2010).

[24] I. Petrov, P. B. Barna, L. Hultman, and J. E. Greene, J. Vac. Sci. Technol. A Vacuum, Surfaces, Film. 21, S117 (2003). 
[25] D. Magnfält, V. Elofsson, G. Abadias, U. Helmersson, and K. Sarakinos, J. Phys. D. Appl. Phys. 46, 215303 (2013).

[26] P. Jensen and B. Niemeyer, Surf. Sci. Lett. 384, L823 (1997).

[27] J. A. Venables, G. D. T. Spiller, and M. Hanbücken, Reports Prog. Phys. 47, 399 (1984).

[28] P. Patsalas and S. Logothetidis, J. Appl. Phys. 93, 989 (2003).

[29] G. S. Bales and A. Zangwill, Phys. Rev. B 55, R1973 (1997).

[30] D. Zhang, L. Guan, Z. Li, G. Pan, X. Tan, and L. Li, Appl. Surf. Sci. 253, 874 (2006).

[31] C. R. Stoldt, C. J. Jenks, P. A. Thiel, A. M. Cadilhe, and J. W. Evans, J. Chem. Phys. 111, 5157 (1999).

[32] S. Stoyanov and I. Markov, Surf. Sci. 116, 313 (1982).

[33] C. Bréchignac, P. Cahuzac, F. Carlier, C. Colliex, J. Leroux, A. Masson, B. Yoon, and U. Landman, Phys. Rev. Lett. 88, 196103 (2002).

[34] P. T. Stroud, J. Phys. C Solid State Phys. 4, 577 (1971).

[35] P. T. Stroud, Thin Solid Films 9, 293 (1972).

[36] Y. L. Lee and J. R. Maa, J. Mater. Sci. 26, 6068 (1991).

[37] M. J. Stowell, Thin Solid Films 21, 91 (1974).

[38] S. Numazawa, M. Ranjan, K.-H. Heinig, S. Facsko, and R. Smith, J. Phys.

Condens. Matter 23, 222203 (2011).

[39] S. Numazawa and R. Smith, Phys. Rev. E. Stat. Nonlin. Soft Matter Phys. 84, 46714 (2011).

[40] H. Brune, K. Bromann, H. Röder, K. Kern, J. Jacobsen, P. Stoltze, K. Jacobsen, and J. Norskov, Phys. Rev. B 52, 14380 (1995).

[41] S. Y. Kim, I.-H. Lee, and S. Jun, Phys. Rev. B 76, 245407 (2007). 
Fig. 1. Experimental data collected for templated films grown at different $F_{T e m p}$ in (a) the coalescence-controlled regime and (b) the coalescence-free regime $\left(F_{T e m p}\right.$ values in the text are given rounded to the second decimal). Horizontal dashed lines indicate $\theta_{\text {Cont }}$ from monolithic films of the different $F_{\text {Temp }}$, coded in color and symbolically. Vertical dash-dotted lines indicate positions of maxima (a)) and minima (b)) taken to signify $\theta_{\text {Elong }}$ for each dataset. (c) Summary of $\theta_{\text {Cont }}$ and $\theta_{\text {Elong }}$ determined in this work together with $\theta_{\text {Perc }}$ from reference 16 . Dashed lines indicate power law fitting of the data points in the two different growth regimes. The numbers indicate fitted slopes, i.e. the $\chi$-value of a $F^{\chi}$ function fitted to the data points.

Fig. 2. Simulated $\theta_{\text {Elong }}$ for two types of templated growth as a function of $\theta_{\text {Temp }}$, with $F_{1}=25 \mathrm{ML} / \mathrm{s}$ and $F_{2}=0.25 \mathrm{ML} / \mathrm{s}$. Horizontal dash-dotted lines indicate $\theta_{\text {Elong }}$ for monolithic films at deposition rates $F_{1}(\sim 1 \mathrm{ML})$ and $F_{2}(\sim 3.3 \mathrm{ML})$. Island densities are shown in the inset for points $A$ (solid) and B (dashed) as functions of the nominal film thickness. 

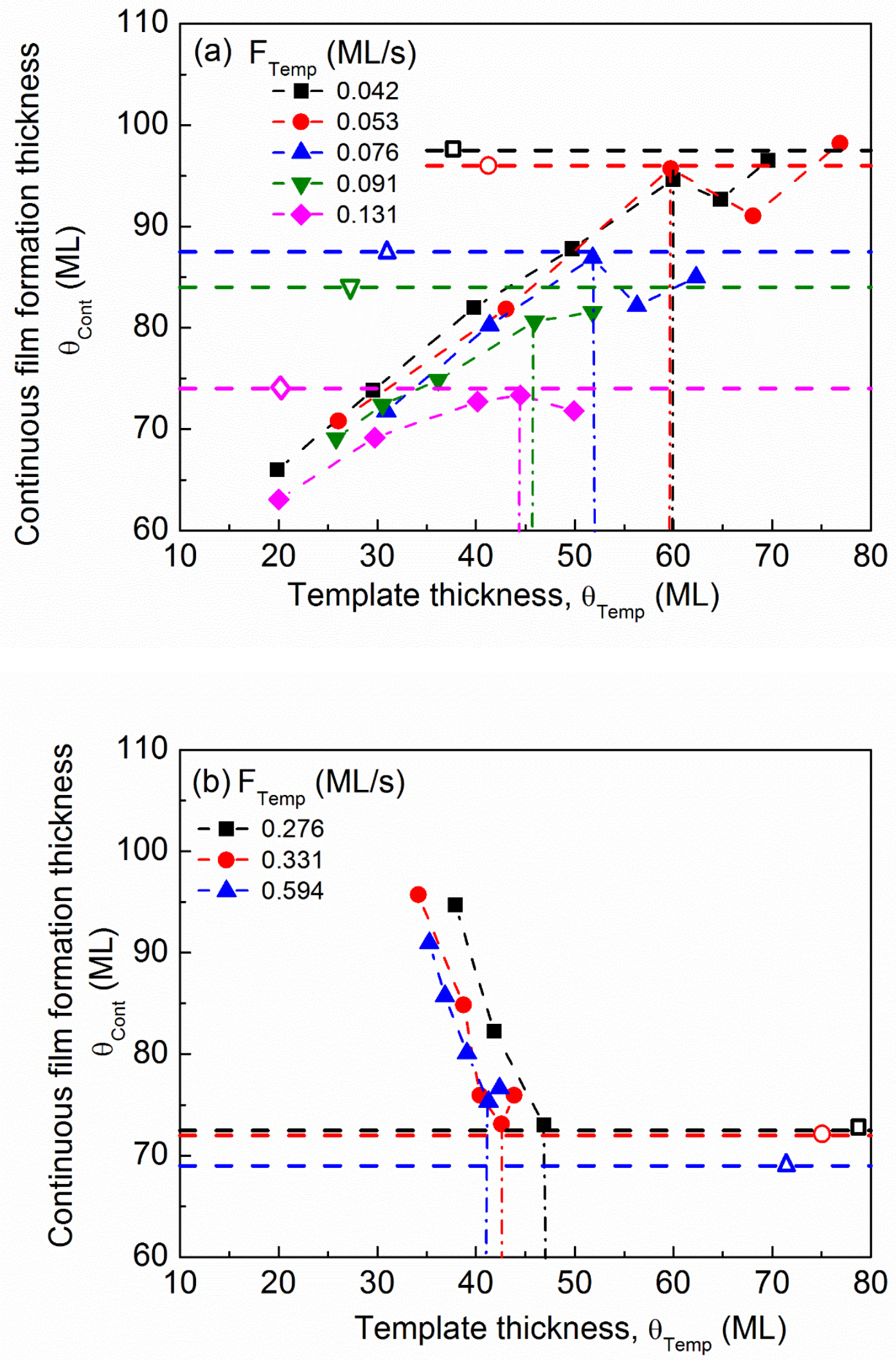


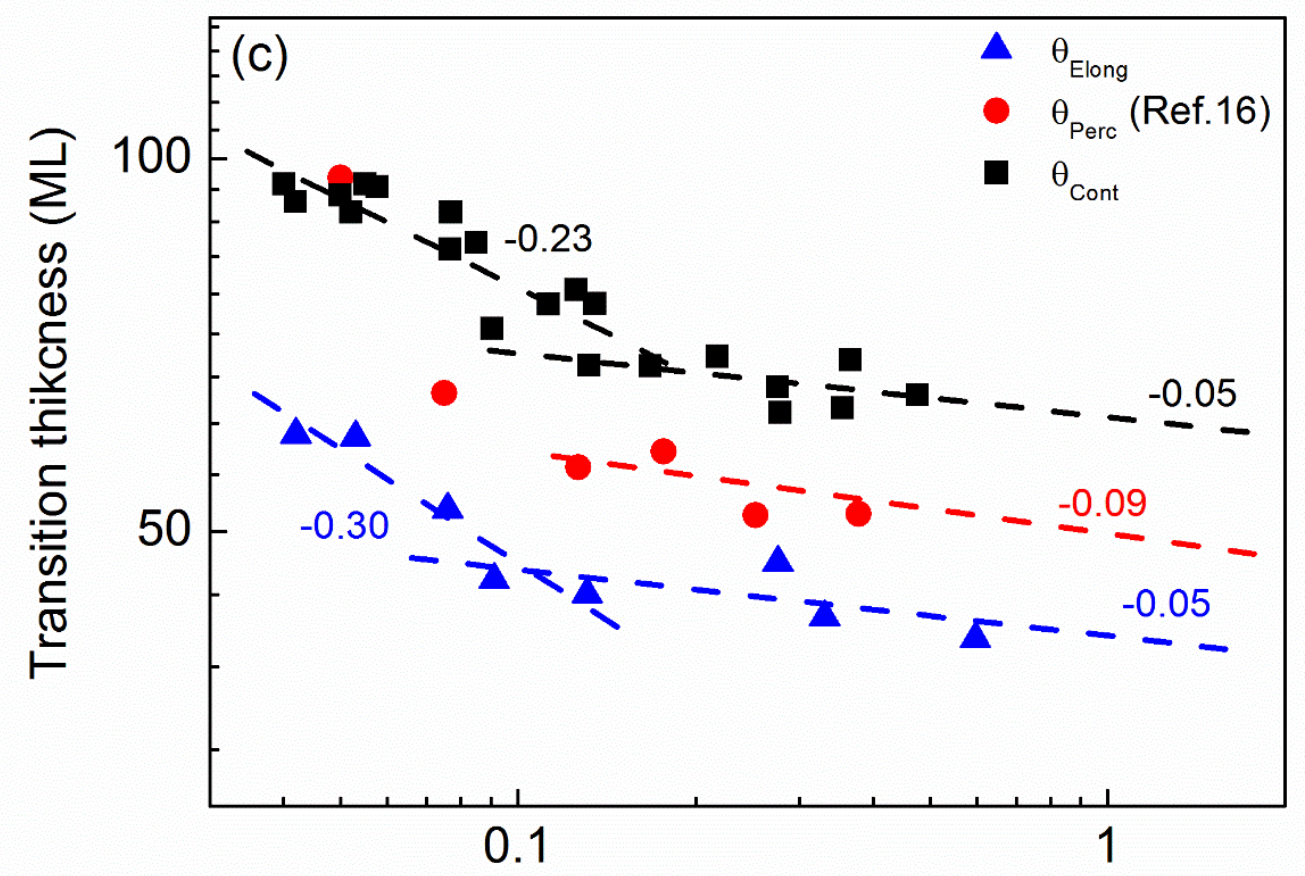

Deposition rate, $\mathrm{F}$ (ML/s)

FIG. 1 


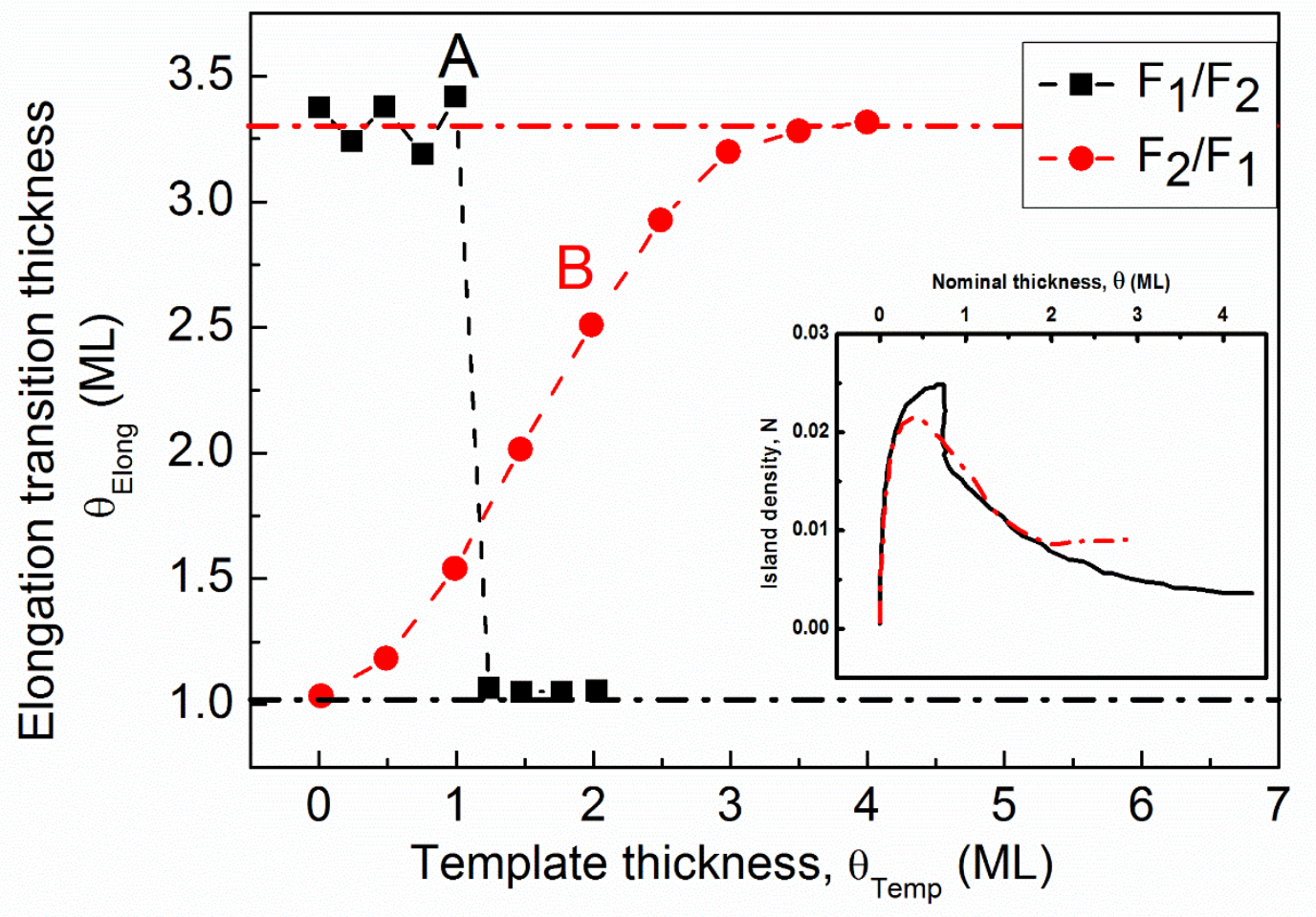

FIG. 2 


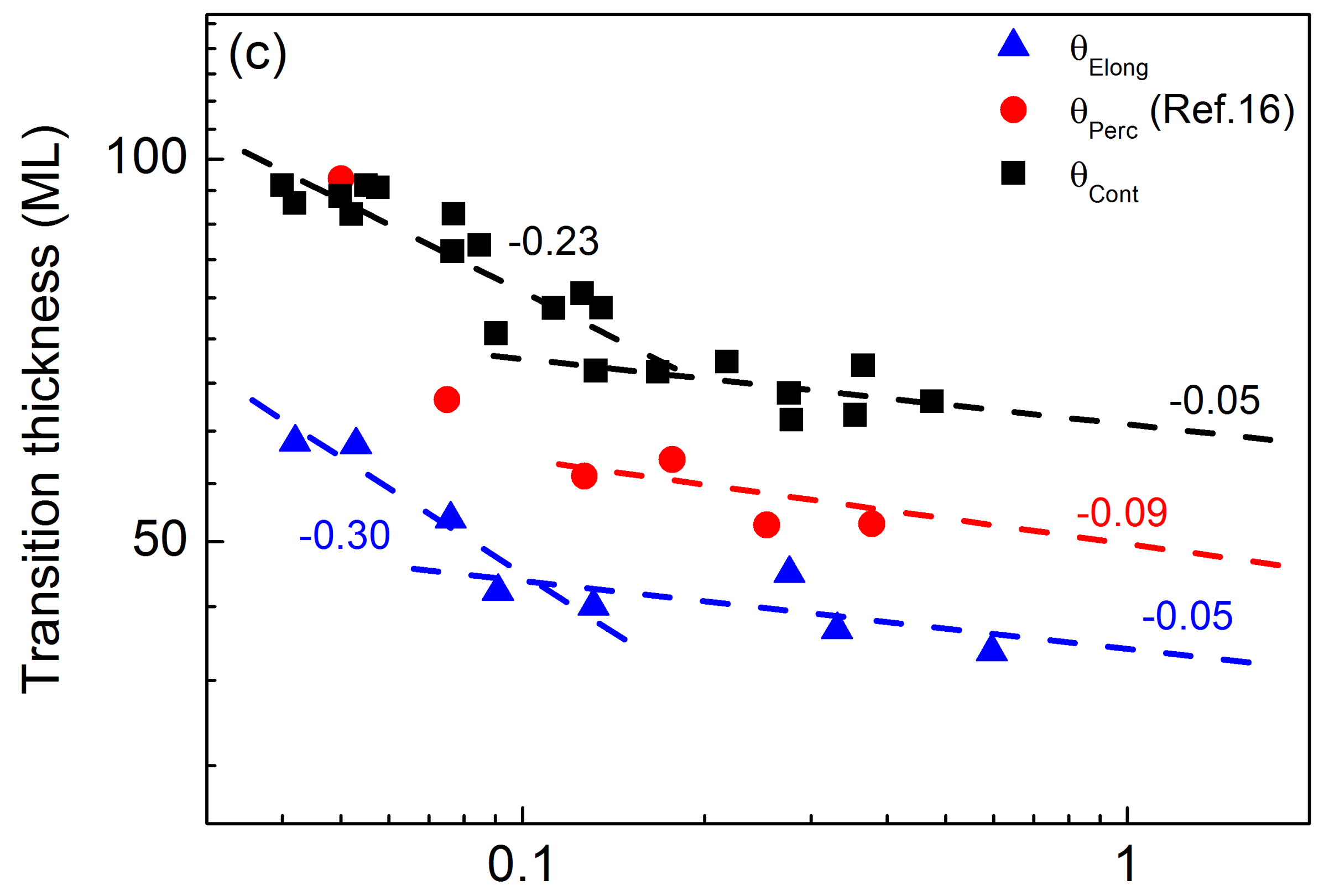

Deposition rate, $\mathrm{F}$ (ML/s) 


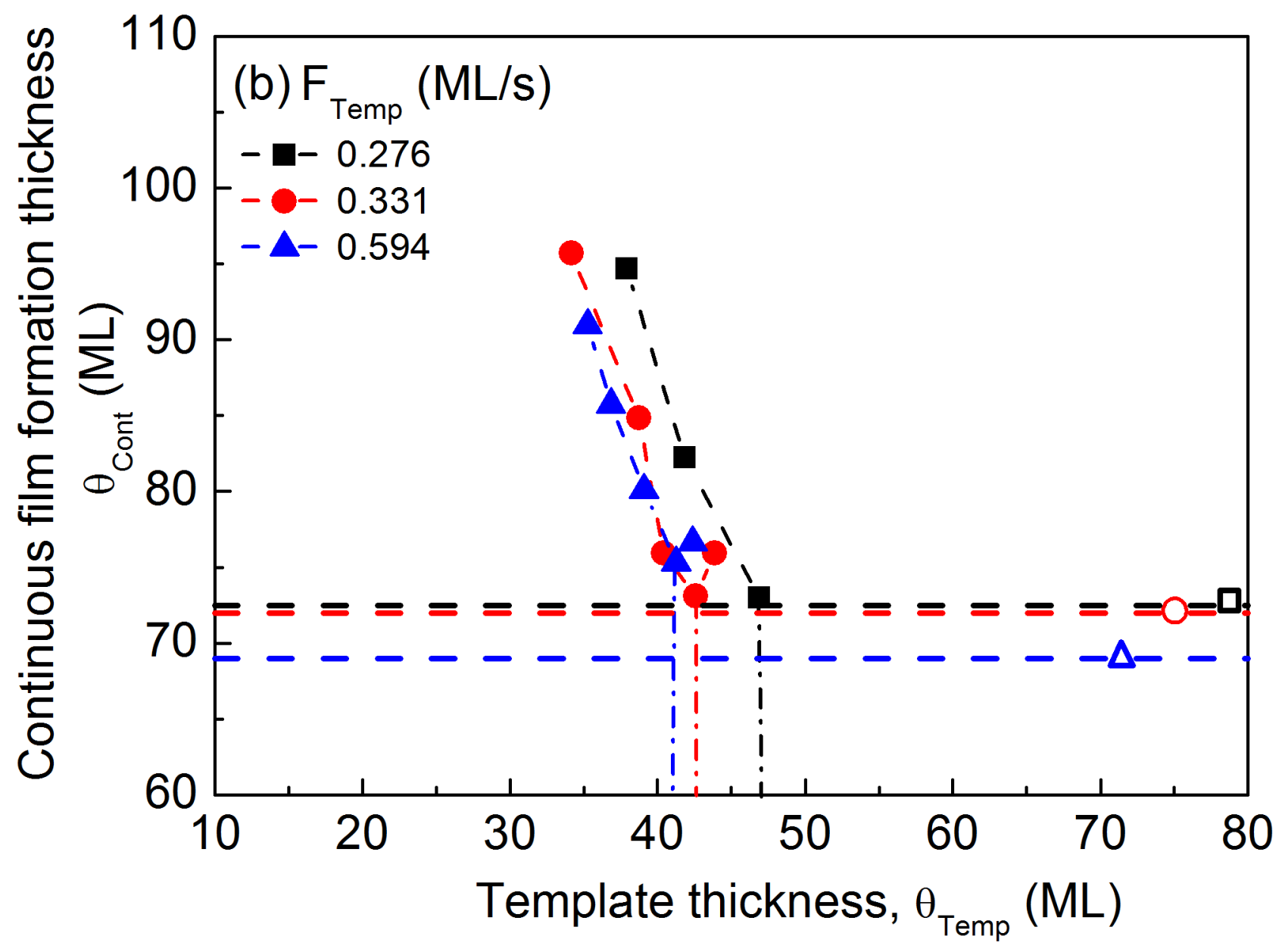




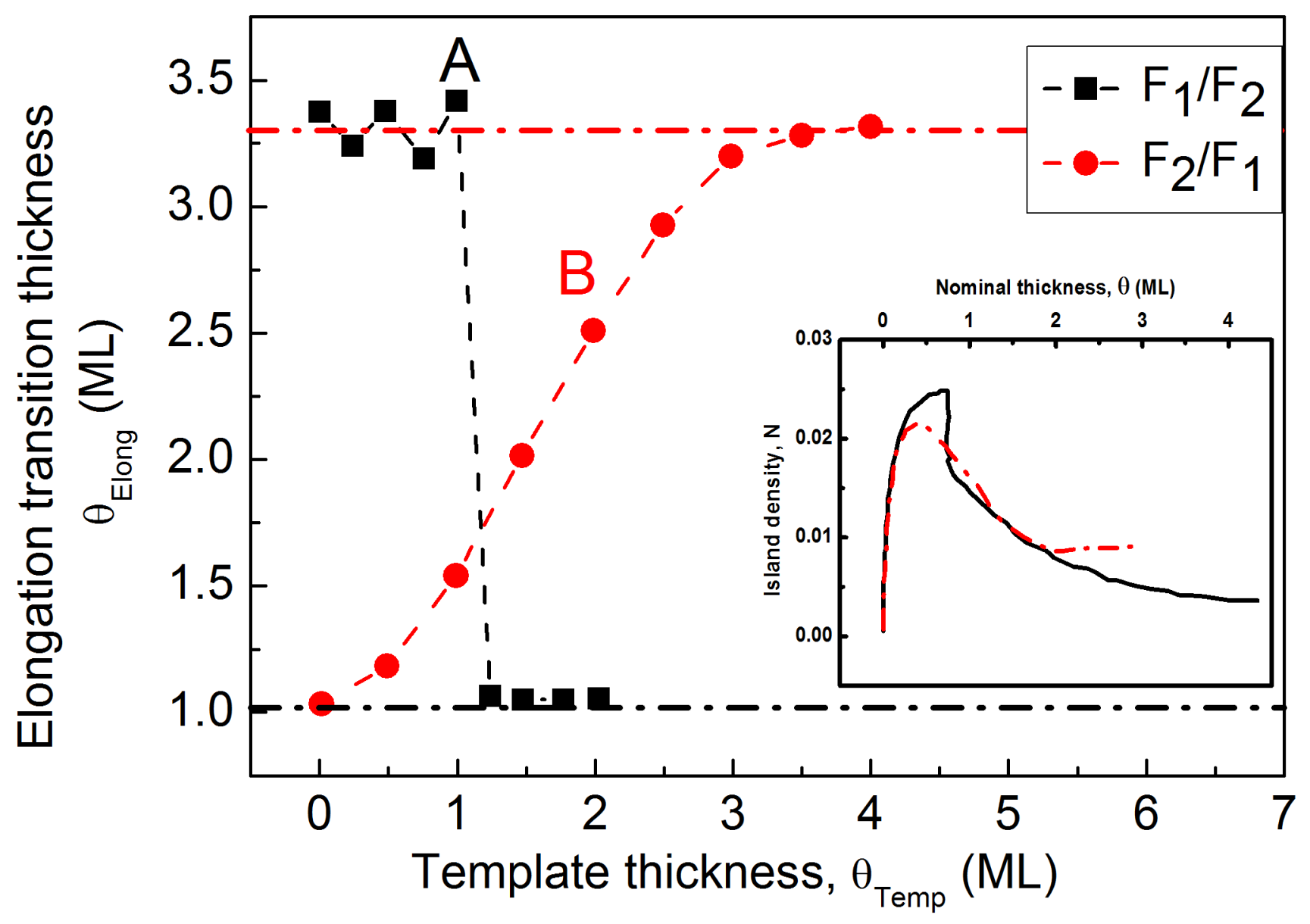

